\title{
Stat 3 protects against Fas-induced liver injury by redox-dependent and -independent mechanisms
}

\author{
Sanae Haga, ${ }^{1}$ Keita Terui, ${ }^{1}$ Hui Qi Zhang, ${ }^{1}$ Shin Enosawa, ${ }^{1}$ Wataru Ogawa, ${ }^{2}$ \\ Hiroshi Inoue, ${ }^{2}$ Torayuki Okuyama, ${ }^{3}$ Kiyoshi Takeda, ${ }^{4}$ Shizuo Akira, ${ }^{4}$ Tetsuya Ogino, ${ }^{5}$ \\ Kaikobad Irani, ${ }^{6}$ and Michitaka Ozaki ${ }^{1,5}$
}

\begin{abstract}
${ }^{1}$ Department of Innovative Surgery, National Research Institute for Child Health and Development, Tokyo, Japan ${ }^{2}$ Division of Diabetes and Digestive and Kidney Diseases, Department of Clinical Molecular Medicine, Kobe University Graduate School of Medicine, Kobe, Japan

${ }^{3}$ Department of Clinical Genetics and Molecular Medicine, National Center for Child Health and Development, Tokyo, Japan ${ }^{4}$ Department of Host Defense, Research Institute for Microbial Diseases, Osaka University, Osaka, Japan

${ }^{5}$ Department of Food and Health Science, Okayama University Graduate School of Medicine and Dentistry, Okayama, Japan

${ }^{6}$ Department of Medicine, Johns Hopkins University School of Medicine, Baltimore, Maryland, USA
\end{abstract}

Signal transducer and activator of transcription-3 (Stat3) is one of the most important molecules involved in the initiation of liver development and regeneration. In order to investigate the hepatoprotective effects of Stat3, we examined whether Stat3 protects against Fas-mediated liver injury in the mouse. A constitutively activated form of Stat 3 (Stat3-C) was adenovirally overexpressed in mouse liver by intravenous injection, and then a nonlethal dose of Fas agonist (Jo2) was injected intraperitoneally into the mouse $(0.3 \mu \mathrm{g} / \mathrm{g}$ body wt). Stat3-C dramatically suppressed both apoptosis and necrosis induced by Jo2. In contrast, liver-specific Stat3-knockout mice failed to survive following Jo2 injection. Stat3-C upregulated expression of FLICE inhibitor protein (FLIP), Bcl-xL, and Bcl-2, and accordingly downregulated activities of FLICE and caspase-3 that were redox-independent. Interestingly, Stat3-C also upregulated the redox-associated protein redox factor-1 (Ref-1) and reduced apoptosis in liver following Jo2 injection by suppressing oxidative stress and redox-sensitive caspase3 activity. These findings indicate that Stat 3 activation protects against Fas-mediated liver injury by inhibiting caspase activities in redox-dependent and -independent mechanisms.

J. Clin. Invest. 112:989-998 (2003). doi:10.1172/JCI200317970.

\section{Introduction}

Signal transducer and activator of transcription-3 (Stat3) is ubiquitously expressed and is transiently activated by a large number of different ligands, including IL-6, leukemia-inhibitory factor, EGF, and PDGF, as well as by a number of oncogenic receptor- and nonreceptor (srclike) tyrosine kinases (1-5). Stat3 was first described as a molecule with DNA-binding activity, found in IL-6-stimulated hepatocytes, that was capable of selectively interacting with an enhancer element in the promoter of

Received for publication January 27, 2003, and accepted in revised form July 29, 2003.

Address correspondence to: Michitaka Ozaki, Department of Innovative Surgery, National Research Institute for Child Health and Development, 3-35-31 Taishi-Do, Setagaya, Tokyo 154-8567, Japan. Phone: 81-3-3416-0181, ext. 8774; Fax: 81-3-3411-7309; E-mail:mozaki@nch.go.jp.

Conflict of interest: The authors have declared that no conflict of interest exists.

Nonstandard abbreviations used: signal transducer and activator of transcription-3 (Stat3); liver-specific Stat3-knockout mice (LS3-KO mice); FLICE inhibitor protein (FLIP); constitutively activated Stat3 (Stat3-C); redox factor-1 (Ref-1); a replicationdeficient adenoviral vector encoding Stat3-C (AxCAS3-C); $\mathrm{N}$-acetyl-L-cysteine (NAC); manganese superoxide dismutase (MnSOD); reactive oxygen species (ROS); physiological saline (PS); glutamic-oxaloacetic transaminase (GOT); glutamic-pyruvic transaminase (GPT). acute-phase genes known as the acute-phase response element $(6,7)$. Stat 3 is activated by the entire family of IL-6-type cytokines, which signal through gp130 and related receptors, and by some growth factors, oncoproteins, and interferons $(8,9)$. The physiological functions of Stat 3 have been extensively studied, and it is known to play crucial roles in the development of various organs and in cell proliferation (5). Though targeted disruption of the Stat 3 gene actually leads to early embryonic lethality (10), studies have been done using mice with conditional knockout (liver, lymphocyte) of Stat3 $(10,11)$. In liver-specific Stat3-knockout mice (LS3-KO mice), no abnormality in liver development and structure was observed, but these mice showed less mitogenic response following partial hepatectomy (11). Also, many other studies have been reported indicating that Stat 3 is involved in several important immediate early response genes following partial hepatectomy $(3,12)$.

In addition to promoting proliferation, Stat 3 also has antiapoptotic properties. When stimulated with serum withdrawal and UV irradiation, Stat 3 competes with the proapoptotic effect of Stat 1 in fibroblasts by inducing expression of Bcl-xL and Survivin (13). Stat 3 may cause cellular proliferation by preventing apoptosis in some types of cells (13-15). However, the precise mechanism for the antiapoptotic property of Stat 3 is not well known. 
Apoptosis is mediated through various biochemical steps. One of the best-characterized apoptotic pathways is that induced through the surface molecule Fas (16, 17). Fas agonist causes trimerization of Fas and recruits a number of molecules to form a complex death-inducing signaling complex (DISC), starting with recruitment of the Fas-adapter protein FADD through Fas and FADD's mutual death domains. The other end of FADD contains two death-effector domains (DEDs) that recruit caspase-8 (FLICE) or its enzymatically inactive homologue, the Fas inhibitory FLICE inhibitor protein (FLIP). Procaspase-8 is cleaved and activated after binding to FADD (18-20). This starts the following cascade reactions toward apoptosis. Interestingly, IL-6, which is known to play a crucial role in liver regeneration via Stat3, protects liver against Fas-mediated apoptosis by inducing a critical level of antiapoptotic proteins such as FLIP, Bcl-2, and Bcl-xL (19).

Clinically, Fas has been shown to be important in hepatitis and other liver diseases (21-23). The liver is very sensitive to Fas-mediated apoptosis because Fas antigen is constitutively expressed on hepatocytes. When mice are injected with anti-Fas antibody, death due to liver failure follows (19). $\mathrm{Fas}^{-/-}$mice show hepatic hyperplasia, suggesting that the Fas pathway may be important for establishing liver structure and size. The Fas/FasL system seems to be involved in many pathological situations, such as graft-versus-host disease, liver transplant, hepatitis B and C, acute alcoholic hepatitis, and some biliary diseases $(21,23,24)$.

The present study was designed to investigate the protective effect of Stat 3 against Fas-mediated liver injury. An adenovirally overexpressed, constitutively activated form of Stat3 (Stat3-C) dramatically suppressed Fasmediated liver injury by upregulating protein levels of FLIP, Bcl-2, Bcl-xL, and redox factor-1 (Ref-1).

\section{Methods}

Adenoviral vectors. The Stat3-C construct, which was provided by James E. Darnell (Rockefeller University, New York, USA), was made by substituting cysteine residues for A661 and N663 of murine Stat 3 and was tagged with FLAG. This renders the Stat3-C molecule capable of dimerizing without a phosphate on Y705 (25). A replication-deficient adenoviral vector encoding Stat3C (AxCAS3-C) was constructed in our laboratory. AdLac $Z$, an adenovirus encoding inert bacterial $\beta$-gal, was used as a control vector. AdRef and AdFer, encoding full-length Ref- 1 in the sense and antisense directions, respectively, have been reported previously (26).

Animal experiments. C57BL/6 male mice, 6-8 weeks old, were used for the experiments. Adenoviral vectors were injected intravenously via tail vein 3 days prior to experiments $\left(3 \times 10^{8} \mathrm{PFU} /\right.$ mouse). In experiments using multiple viral vectors, total amounts of adenoviruses injected were adjusted to $3 \times 10^{8} \mathrm{PFU} /$ mouse. After a 24hour fast, the mice were injected intraperitoneally with Jo2 antibody $(0.3 \mu \mathrm{g} / \mathrm{g}$ body wt; BD PharMingen, San Diego, California, USA). In some experiments, $N$-acetyl-
L-cysteine (NAC) $(200 \mathrm{mg} / \mathrm{kg}$ body wt) (27) and the general apoptosis inhibitor Z-VAD-fmk $(1 \mu \mathrm{g} / \mathrm{g}$ body wt; Enzyme Systems Products Inc., Livermore, California, USA) (28) were intraperitoneally injected 1 hour prior to the experiment. All animals were handled according to the policies set by the Laboratory Animal Care and Use Committee of The National Research Institute for Child Health and Development.

Experiments using primary cultured hepatocytes. Primary cultured hepatocytes were prepared by a conventional collagenase perfusion method from C57BL/6 mouse liver. Hepatocytes were seeded at $3 \times 10^{6}$ cells per $10-\mathrm{cm}$ dish 24 hours prior to adenoviral infection. AdLacZ or AxCAS3-C was infected at an MOI of 2 , followed by Jo2 administration at $2.5 \mu \mathrm{g} / \mathrm{ml} 48$ hours later. Cells were harvested for the analysis of protein expression 48 hours after adenoviral infection, and apoptosis assays were performed 8 hours after Jo2 administration.

Generation of LS3-KO mice. We generated LS3-KO mice by breeding Stat3-flox mice (15) with albumin-Cre transgenic (Alb-Cre) mice (29), which express Cre recombinase specifically in the liver under the control of the albumin promoter. To increase the efficiency of Stat 3 disruption, we also introduced a Stat 3 null allele over the floxed allele by crossing with Stat 3 heterozygous knockout $\left(\mathrm{Stat}_{3}^{+-}\right.$) mice (10). We crossed Stat3 $3^{+-}$ mice with Alb-Cre mice and generated Stat $3^{+/}$, Alb-Cre mice, then bred these mice with mice harboring homozygous Stat 3 floxed allele (Stat3floxfflox). The resulting Stat $3^{f l o x /}$, Alb-Cre mice were used as LS3-KO mice. Stat $3^{f l o x}$ - mice obtained from the same breeding were used as control mice.

Western blot analysis. The liver homogenates were centrifuged and the supernatants were used for the assay. Fifty micrograms of protein was used for $15 \%$ SDSPAGE. The protein transferred to the nitrocellulose membrane was incubated with the proper antibodies, anti-Fas, anti-Bcl-2, anti-Bcl-xL, anti-Survivin (Santa Cruz Biotechnology Inc., Santa Cruz, California, USA), anti-FLIP-CT (Cell Signaling Technology Inc., Beverly, Massachusetts, USA), anti-Ref-1, anti-manganese superoxide dismutase (anti-MnSOD; Transduction Laboratories, San Diego, California, USA), anti-thioredoxin (BD PharMingen), and anti-FLAG (SigmaAldrich, St. Louis, Missouri, USA).

Electrophoretic mobility shift assay. Stat3 DNA binding activity was assayed using the SIE-m67 oligonucleotide as a probe $\left(5^{\prime}\right.$-actgGGATTTTTCCCGTAAATGGTC- $\left.3^{\prime}\right)$. The reaction mixture contained nuclear protein extract $(5$ $\mu \mathrm{g})$, DTT $(2 \mathrm{mM})$, dI-dC $(2 \mu \mathrm{g})$, ssDNA $(10 \mu \mathrm{g} / \mathrm{ml})$, and ${ }^{32} \mathrm{P}$-labeled SIE-m67 probe $\left(5 \times 10^{5} \mathrm{cpm}\right)$. The specimens were electrophoresed on $5 \%$ polyacrylamide native gels at $4^{\circ} \mathrm{C}$ in $0.25 \times$ Tris-Borate/EDTA (TBE) buffer.

Histological studies. Liver tissue was excised and fixed with $10 \%$ buffered formalin, and paraffin-embedded sections were stained with H\&E. For immunohistochemical demonstration of Stat 3 protein, specimens were incubated with primary antibody (anti-Stat3; BD Transduction Laboratories, Lexington, Kentucky, USA) 


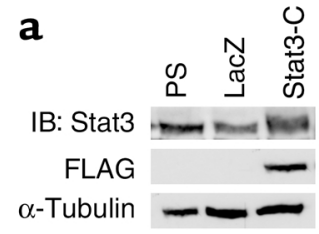

b
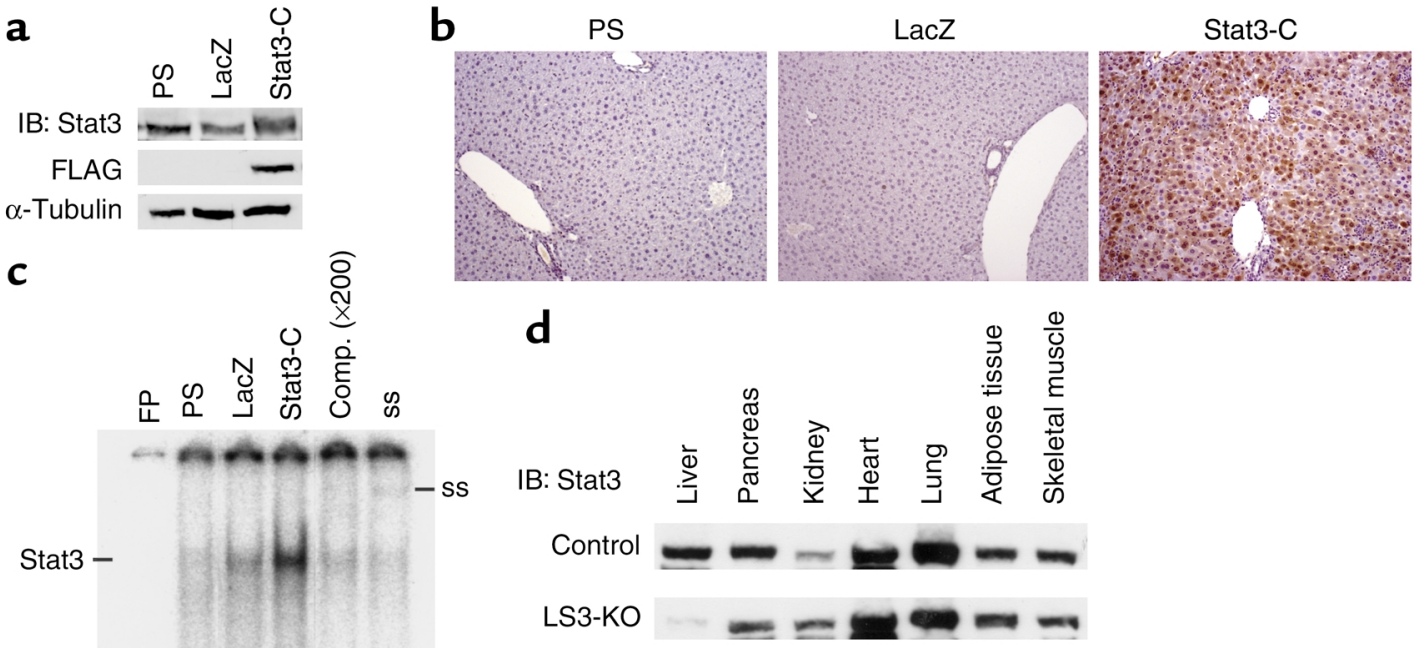

\section{Figure 1}

Stat3-C protein is successfully overexpressed in mouse liver and activates Stat3 DNA binding. (a) AntiStat 3 and anti-FLAG immunoblots were performed to determine total and exogenous Stat3 protein in liver tissue, respectively, 3 days after adenovirus infection. (b) Immunohistochemical study using anti-Stat3 showed that Stat3-C protein is expressed in nuclei and cytoplasm of the liver in Stat3-C mice. Original magnification, $\times 200$. (c) Electrophoretic mobility shift assay (EMSA) analysis showed elevated Stat3 DNA binding in the liver of Stat3-C mice. Five micrograms of nuclear extract was incubated with SIE-m67 probe. In order to confirm specific Stat3 DNA binding, competition (comp.) and supershift assays were performed using cold SIE-m67 probe and anti-Stat3. (d) Western blot analysis of Stat 3 protein in various tissues of control and LS3-KO mice. These data are representative of at least three independent experiments. FP, free probe; ss, supershift; IB, immunoblot.

at $2.5 \mu \mathrm{g} / \mathrm{ml}$ at $4^{\circ} \mathrm{C}$ overnight, followed by the secondary antibody (biotin-conjugated anti-mouse IgG).

Apoptosis assay. The In situ Cell Death Detection Kit (Roche Diagnostics Corp., Basel, Switzerland) was used for the demonstration of apoptotic cell death of the liver tissue. Paraffin-embedded liver specimens were incubated with the TUNEL reaction mixture according to manufacturer's recommendations. Also, Cell Death Detection ELISA PLUS (Roche Diagnostics Corp.) was used for the quantitative evaluation of apoptotic cells. Lysates of liver tissue were directly applied for this assay ( $40 \mu \mathrm{g}$ of protein), and the assay was performed according to the manufacturer's recommendations.

Caspase activity assay. Activities of caspase- $3,-8$, and -9 were measured using the CPP32/Caspase- 3 Colorimetric Protease Assay Kit, FLICE/Caspase-8 Colorimetric Protease Assay Kit, and Caspase-9/Mch6 Colorimetric Protease Assay Kit, respectively, from Medical and Biological Laboratories Co. (Tokyo, Japan). Supernatant of liver homogenate was prepared $(200 \mu \mathrm{g}$ in $50 \mu \mathrm{l})$ and reacted with the substrate at $37^{\circ} \mathrm{C}$ for 1 hour prior to the assay.

Hepatic reactive oxygen species assay in liver tissue. Production of reactive oxygen species (ROS) in liver tissue was measured by two independent methods, lucigeninenhanced chemiluminescence and hydrogen peroxide assays, the details of which were described previously (30). After isolating hepatocytes by conventional perfusion methods, $100 \mu \mathrm{l}$ of cell suspension $\left(10^{5}\right.$ cells $)$ was added to $1 \mathrm{ml}$ of lucigenin buffer $(75 \mathrm{mM}$ luci- genin in HBSS). The chemiluminescent signal was monitored every 5 minutes for 30 minutes. The emitted light units (after subtracting a blank and integrating over 15 minutes) were used as a measure of superoxide production. Hydrogen peroxide production was measured using the Amplex Red Hydrogen Peroxide Assay Kit (Molecular Probes Inc., Eugene, Oregon, USA). Supernatant of liver homogenate was used for the assay, according to the manufacturer's recommendations. After subtraction of a blank sample, the red fluorescence signal was standardized by protein concentration, and hydrogen peroxide concentration (expressed as $\mathrm{mmol} / \mathrm{mg}$ protein) was calculated based on a standard curve.

Analysis of $m R N A$. For multiprobe ribonuclease protection assay, the presence of transcripts of the indicated genes and the internal control $L 32$ were detected using a multiprobe template set (BD PharMingen). Probe synthesis, hybridization, and RNase treatment were performed according to the manufacturer's recommendations. The protected transcripts were resolved by electrophoresis on denaturing $4.75 \%$ polyacrylamide gels and quantified on a PhosphorImager (Amersham Biosciences, Piscataway, New Jersey, USA). For Northern blot analysis, total cellular RNA was extracted and electrophoresed on a $1.4 \%$ agarose gel. RNA was transferred to a nylon membrane, which was hybridized with $3 \times 10^{7} \mathrm{cpm}$ of ${ }^{32}$ P-labeled single-strand Ref- 1 cDNA. After washing, the membrane was autoradiographed. 
Transient transfection experiments and luciferase reporter assay. The human Ref- 1 promoter was cloned by PCR from HeLa genomic DNA as previously reported (31, 32) and cloned into pGL3 (Promega Corp., Madison, Wisconsin, USA). HeLa cells were seeded at a density of $1.5 \times 10^{4} / \mathrm{cm}^{2}$, and LipofectAMINE (Invitrogen Corp., Carlsbad, California, USA) was used as the transfectant according to the manufacturer's recommendations. Forty-eight hours after transfection of Ref-1 promoter gene, adenoviral vector was infected at various MOI as indicated. Luciferase activity was assessed 36 hours after adenovirus infection using the Bright-Glo luciferase assay system (Promega Corp.) according to the manufacturer's recommendations. Results of each reporter assay were reproducible in three separate experiments.

Statistical analysis. Fisher's test was used for analysis of differences between multiple groups and was considered significant at the 95\% confidence level. The number of animals $(n)$ used for each experiment is indicated in parentheses.

\section{Results}

Expression and activity of Stat 3 in liver of mice injected with AxCAS3-C and of LS3-KO mice. DNA binding. Adenoviral vector $\left(1 \times 10^{8} \mathrm{PFU} / \mathrm{mouse}\right)$ encoding a constitutively activated form of the Stat3 gene (AxCAS3-C) was intravenously injected into mice 3 days prior to the experiment. This successfully overexpressed a constitutively activated form of Stat3 protein (Stat3-C) in mouse liver (Figure 1, a and b). Immunoblot and immunohistochemical analyses using anti-Stat 3 demonstrated that the amount of total Stat3 protein in the liver of mice infected with AxCAS3-C (Stat3-C mice) was higher than that in mice injected with physiological saline (PS mice) and AdLacZ (LacZ mice). The expres-

\section{Figure 2}

Stat3-C prevents liver injury induced by Fas agonist. (a) Survival after Fas agonist treatment. Mice were injected intraperitoneally with Jo2 $(0.3 \mu \mathrm{g} / \mathrm{g}$ body wt) and followed for 72 hours. (b) Liver appearance and microscopic change 6 hours after Fas agonist. H\&E staining; original magnification, $\times 100$. (c) Serum levels of GOT and GPT (sGot and sGPT) until 72 hours after Fas agonist treatment. ${ }^{*} P \leq 0.01$ vs. LacZ 24 hours after treatment with Fas agonist. Data are expressed as mean $\pm \operatorname{SEM}(n=5)$.

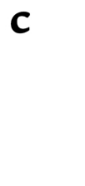

sion of exogenously expressed Stat $3-C$ protein was also confirmed by anti-FLAG in the liver of Stat3-C mice (Figure 1a). Immunohistochemical analysis revealed that Stat3-C protein was expressed in both the cytoplasm and nucleus in the liver of Stat3-C mice, though Stat 3 protein was hardly detected in the liver of PS and LacZ mice (Figure $1 b)$. Stat $3-C$ resulted in no abnormality observed in the hepatic structure.

Stat3 DNA binding was not activated in the PS mouse liver in quiescent conditions. Overexpression of Stat3-C resulted in strong activation of its DNA binding, whereas AdLacZ infection only partially activated Stat3 DNA binding (Figure 1c).

In the liver of LS3-KO mice, the amount of Stat3 protein was less than $5 \%$ of that of Stat $3^{\text {flox } /-}$ mice, whereas various other tissues including pancreas, kidney, heart, lung, adipose tissue, and skeletal muscle did not differ in Stat3 expression between control and LS3-KO mice (Figure 1d).

Stat3-C prevents liver injury induced by Fas agonist. Expression of Stat3-C resulted in a liver that was macroscopically normal in size and color with microscopic mild
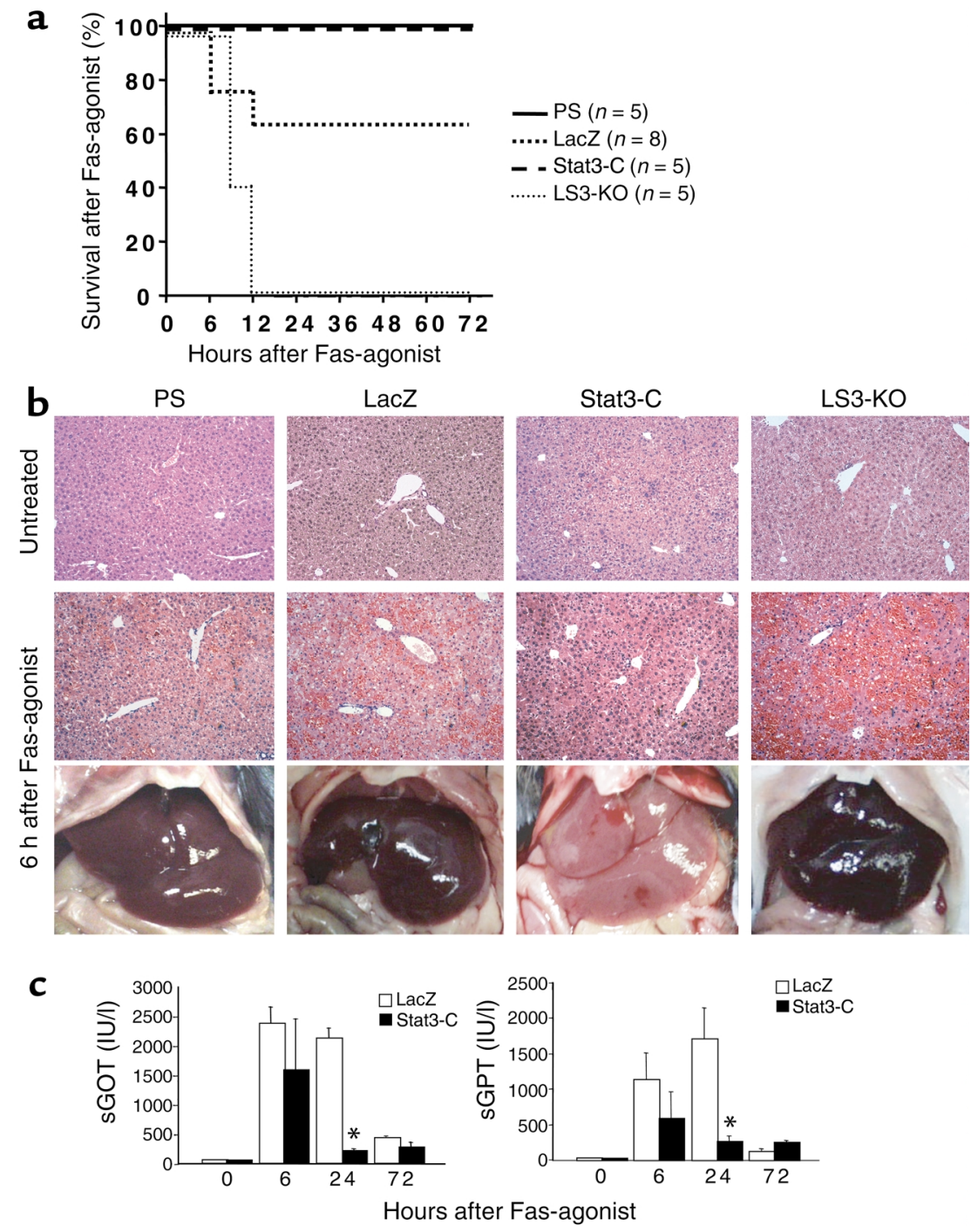
a
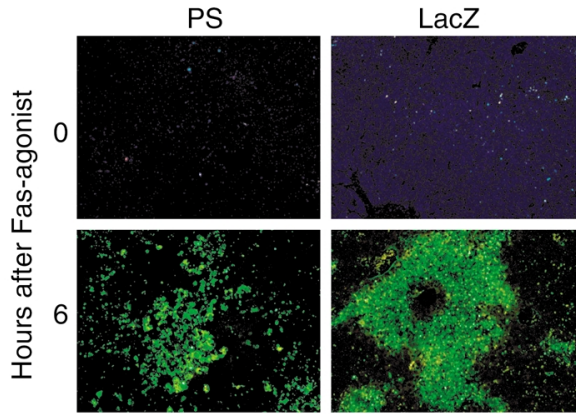

b

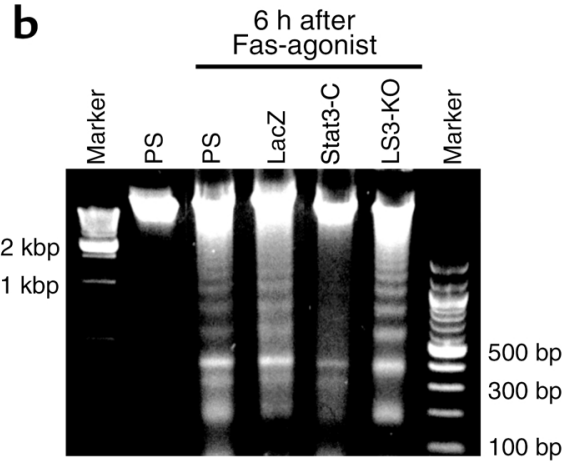

d

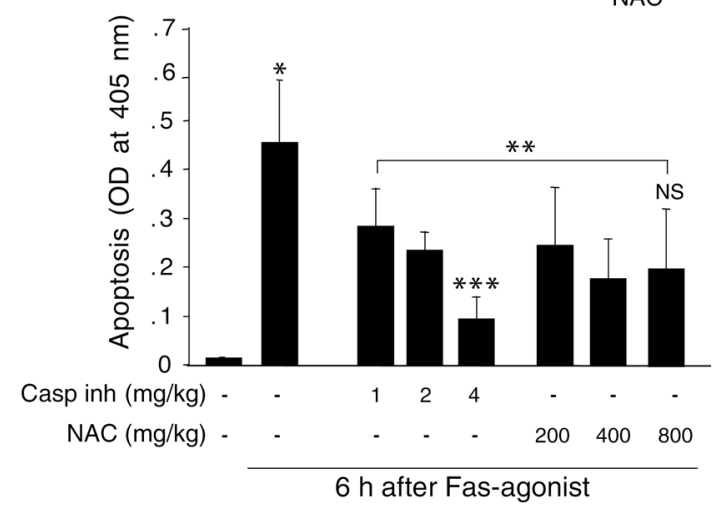

Stat3-C
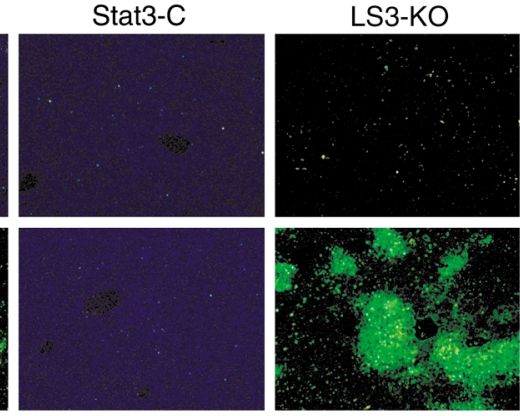

c

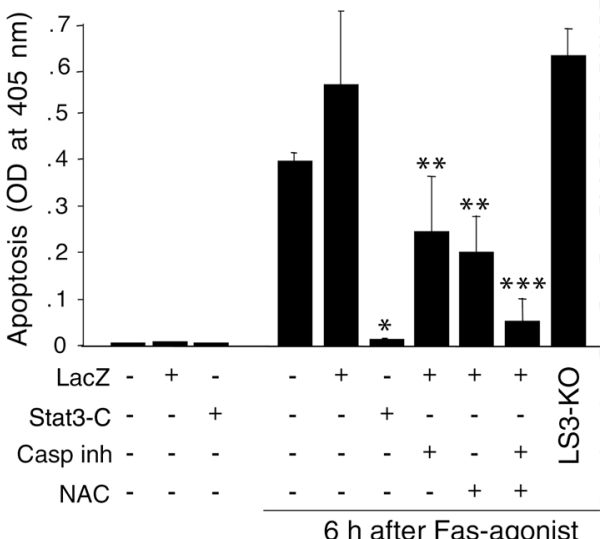

$6 \mathrm{~h}$ after Fas-agonist
Figure 3

Stat3-C suppresses Fas-induced apoptotic cell death in liver. (a) Fluoromicroscopic photographs of liver sections with TUNEL staining from PS, LacZ, Stat3-C, and LS3-KO mice treated with Fas agonist (formalin-fixed, paraffin-embedded sections; original magnification, $\times 100)$. (b) The DNA ladder pattern was analyzed 6 hours after Fas agonist. Each photo is representative of at least three independent experiments. (c) Apoptotic cell death was quantitatively measured by ELISA. Pretreatment with the general caspase inhibitor Z-VAD-fmk $(1 \mu \mathrm{g} / \mathrm{g}$ body $\mathrm{wt})$ or the antioxidant NAC $(200 \mathrm{mg} / \mathrm{kg}$ body wt) was performed 1 hour prior to the experiments. ${ }^{*} P<0.01$ vs. LacZ/Fas agonist, ${ }^{*} P<0.05$ vs. LacZ/Fas agonist and Stat3-C/Fas agonist, $* * * P<0.05$ vs. LacZ/Fas agonist + caspase inhibitor and LacZ/Fas agonist + NAC. (d) Dose-dependent effects of caspase inhibitor (Z-VAD-fmk) or antioxidant (NAC) upon hepatic apoptosis after Fas agonist. ${ }^{*} P<0.01$ vs. untreated control, ${ }^{*} P<0.05$ vs. Fas agonist, $* * * P<0.05$ vs. Fas agonist + caspase inhibitor ( 1 and $2 \mathrm{mg} / \mathrm{kg}$, respectively). All data are expressed as mean $\pm \operatorname{SEM}(n=5)$. Casp inh, caspase inhibitor. inflammatory cell infiltration 3 days after viral injection. Intraperitoneal injection of Jo2 at $0.3 \mu \mathrm{g} / \mathrm{g}$ body wt induced severe liver injury, but did not show lethality until 72 hours after injection in PS mice and Stat3-C mice. Though three of eight LacZ mice could not survive over 12 hours, LS3-KO mice showed absolute lethality at 12 hours after Jo2 injection (Figure 2a).

At 6 hours following Jo2 injection, the liver turned dark red in color, though the mice were still alive. Histological examination revealed massive hepatic hemorrhage with necrosis at 6 and 24 hours after injection and severe neutrophilic infiltration at 72 hours after injection (Figure 2b). Stat3-C expression markedly reduced Fas-mediated liver injury. Liver was architecturally preserved with sporadic reddish changes in appearance, and microscopically much less bleeding and necrosis were observed 6 hours and 24 hours after Jo2 injection.

Serum levels of glutamic-oxaloacetic transaminase (GOT) and glutamic-pyruvic transaminase (GPT) were remarkably raised 6 and 24 hours after Jo2 injection in LacZ mice. This increase, however, was dramatically reduced in Stat3-C mice (Figure 2c). These data clearly demonstrate that Stat3-C confers resistance against Fas-mediated liver injury.

Stat3-C prevents apoptosis induced by Fas agonist. To confirm antiapoptotic properties of Stat3, hepatic apoptosis induced by Jo2 was studied by three independent methods: TUNEL staining of histological sections, DNA ladder assay, and ELISA (Figure 3, a-c). Massive apoptotic cell death observed 6 hours after Jo2 injection in livers of PS, LacZ, and LS3-KO mice was dramatically reduced by Stat3-C expression. A typical DNA ladder pattern was also identified in specimens from PS, LacZ, and LS3-KO mice (Figure 3b).

Interestingly, pretreatment with the general caspase inhibitor Z-VAD-fmk $(1 \mu \mathrm{g} / \mathrm{g})$ or the antioxidant NAC $(200 \mathrm{mg} / \mathrm{kg})$ inhibited Jo2-induced hepatic apoptosis significantly, but failed to inhibit it completely even with increased amounts of these reagents (Figure 3, c and d). However, when administered together, they suppressed apoptosis more effectively (Figure 3c), with a synergistic suppressive effect almost equal to that of 
Stat3-C. These facts may indicate that activated Stat3 suppresses Fas-induced liver injury via caspase-dependent and/or redox-dependent mechanisms.

Antiapoptotic proteins are induced in liver expressing Stat3-C. To address the antiapoptotic mechanism of Stat 3 acting upon Fas-mediated liver injury, we first investigated the expression of apoptosis-related proteins (Figure 4a).
Overexpressed Stat3-C did not suppress hepatic Fas expression, but upregulated it. Infection with AdLacZ, however, induced Fas to the same degree as was observed in Stat3-C mice. Stat3-C upregulated Bcl family proteins with antiapoptotic properties $(\mathrm{Bcl}-\mathrm{xL}$ and $\mathrm{Bcl}-2)$, whereas adenovirus itself did not induce these proteins. Jo2 injection induced Fas and Bcl-xL proteins

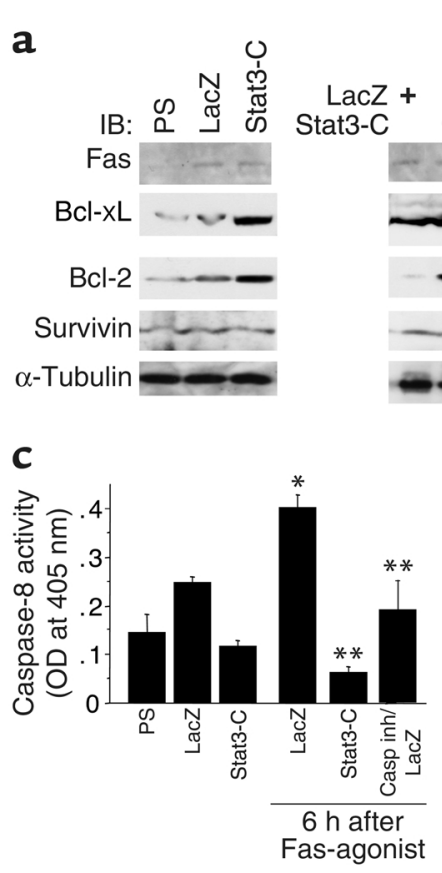

Fas-agonist
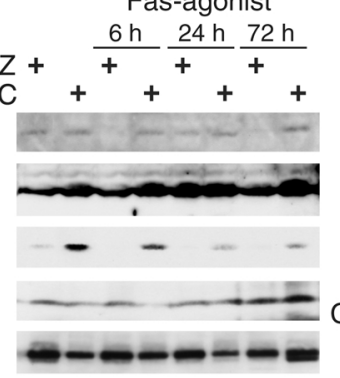

b

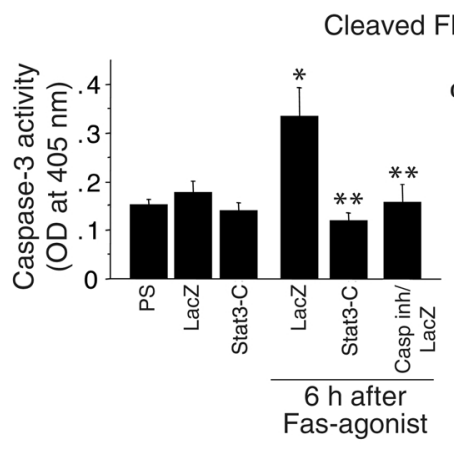

Fas-agonist

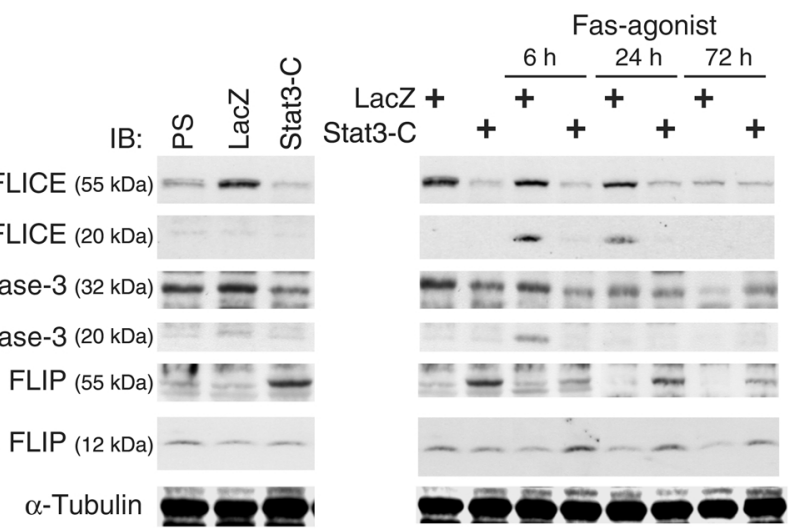

d

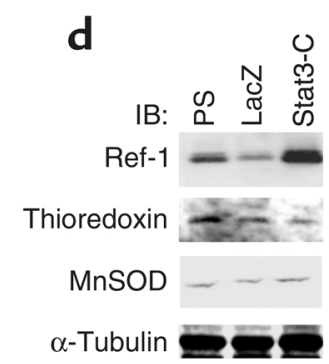

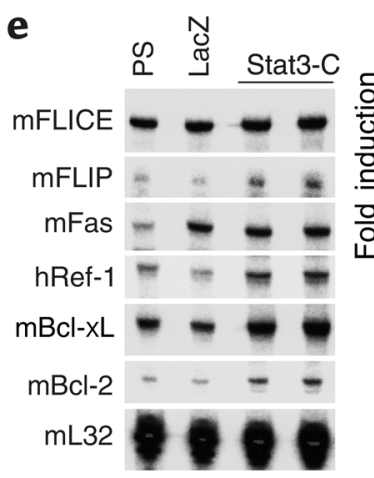

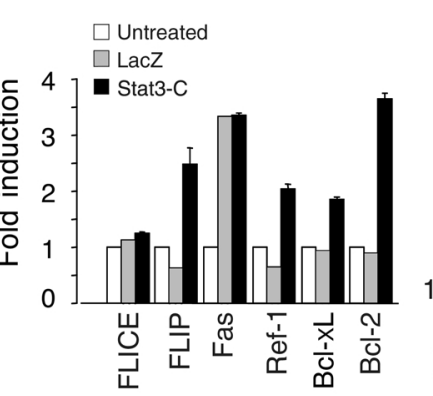

f

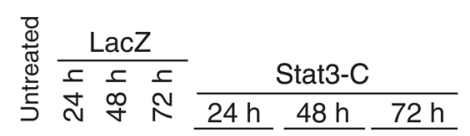
Ref-1 18s rRNA
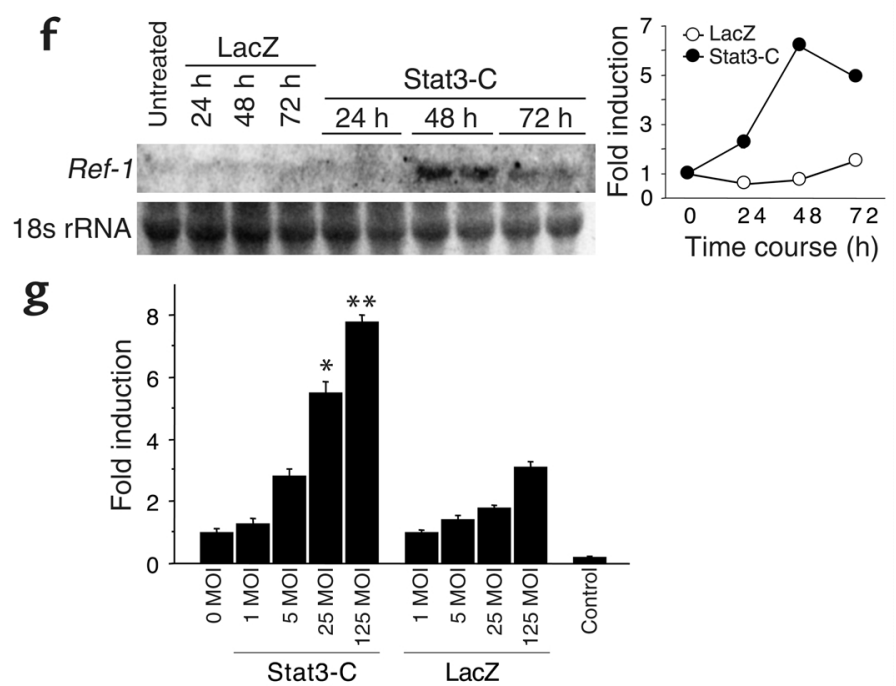

Figure 4

Stat3-C induces apoptosis-related proteins and redox-related protein. Immunoblot, Northern blot, and multiprobe ribonuclease protection assay were performed on liver tissues from PS, LacZ, and Stat3-C mice. Sequential changes of the protein amounts after Fas agonist treatment were analyzed in LacZ and Stat3-C mice. Each blot is representative of three independent experiments. (a) Expression of Fas, Bcl-related proteins, and Survivin. (b) Expression of caspase-related proteins. (c) Enzyme activity assays of caspase-3 and caspase-8. ${ }^{*} P<0.05$ vs. LacZ, ${ }^{*} P<0.01$ vs. LacZ/Fas agonist. (d) Protein expression of redox-related proteins Ref-1, thioredoxin, and MnSOD. (e) Multiprobe ribonuclease protection assay for apoptosis-related genes and redox-related genes 48 hours after adenoviral gene transfer. $\mathrm{m}$, mouse; h, human. (f) Northern blot analysis for Ref-1 mRNA after adenoviral gene transfer of Stat3-C. Results are from a single representative experiment that was reproduced once (d-f). (g) Transcriptional activity of Ref-1 promoter by Stat3-C. Luciferase assay for Ref-1 promoter activity stimulated by AxCAS3-C or AdLacZ. Control was $P G L 3$ without Ref- 1 promoter. ${ }^{*} P \leq 0.01 \mathrm{vs}$. uninfected $(0 \mathrm{MOI}, 0)$ and LacZ $(25 \mathrm{MOI})$, ${ }^{*} P \leq 0.01$ vs. uninfected $(0 \mathrm{MOI})$ and LacZ $(125 \mathrm{MOI})$. All data are expressed as mean $\pm \operatorname{SEM}(n=5)$. 


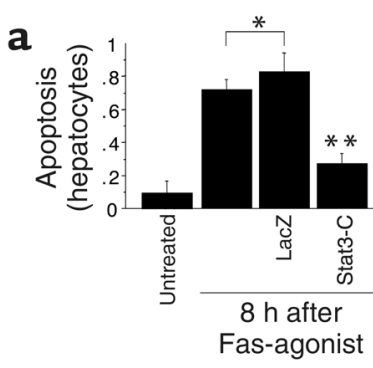

b

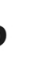

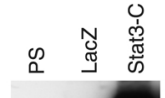

FLAG

Stat3

Ref-1

FLIP

Bcl-2

$\mathrm{BCl}-\mathrm{XL}$

$\alpha$-Tubulin

24 hours after injection in LacZ mice. Stat3-C did not alter the expression of Survivin protein, which is reported to be induced by Stat 3 in some types of cells (13).

FLIP protein is induced and activated by Stat3-C followed by reduction of FLICE and caspase-3 activities. Stat3-C suppressed expression of FLICE protein as well as caspase3 (albeit to a lesser extent), as determined by comparing Stat3-C mice with PS and LacZ mice. Though FLICE and caspase- 3 were both processed and activated 6 hours after Jo2 injection in LacZ mice, Stat3-C inhibited both expression and activation of these proteins by Fas agonist (Figure $4, b$ and $c$ ). FLIP protein was also induced by Stat3-C, but not by PS and LacZ, and was significantly activated following Jo2 injection until 72 hours afterward (Figure 4b). IL-6, one of the major ligands for Stat3, has already been reported to upregulate FLIP, Bcl-2, and Bcl-xL in liver (19). Interestingly, Stat3-C suppressed caspase activity to the same degree as caspase inhibitor did, if not more (Figure 4c). These data may suggest that Stat3 plays a critical role in IL-6associated antiapoptotic activity in liver.

\section{Figure 6}

Hepatic ROS production and apoptosis induced by Fas agonist are suppressed by Stat3-C via Ref-1. LacZ, Stat3-C:LacZ, and Stat3-C:AdFer (Stat3-C:Fer) mice were infected with AdLacZ only, AxCAS3-C:AdLacZ (1:1), and AxCAS3-C:AdFer (1:1), respectively. Total amounts of adenovirus vectors were adjusted to $3 \times 10^{8}$ PFU/mouse in all experiments. (a) Immunoblots of Ref- 1 and Stat 3 proteins showing that AxCAS3-C increased Stat 3 and Ref- 1 proteins, but AdFer reduced them. Ref- 1 protein was, however, expressed even in the liver of LS3-KO mice. (b) Superoxide generation assay and hydrogen peroxide assay in liver tissue ${ }^{*} P<0.05$ vs. LacZ, ${ }^{*} P<0.05$ vs. LacZ/Fas agonist, ${ }^{* *} P<0.05$ vs. Stat3-C:LacZ/Fas agonist. Casp inh; i.e. Z-VAD-fmk. (c) Apoptotic cell death was quantitatively analyzed by ELISA. ${ }^{*} P<0.01$ vs. LacZ, ${ }^{*} P<0.05$ vs. LacZ/Fas agonist, ${ }^{*}{ }^{*} P<0.05$ vs. Stat3-C:LacZ/Fas agonist. (d) Enzyme activity assays of caspase- 3 and caspase- 8 . ${ }^{*} P<0.05$ vs. LacZ/Fas agonist. All data are expressed as mean $\pm \operatorname{SEM}(n=5)$. RLU, relative light unit.

\section{Figure 5}

Stat3-C protects primary hepatocytes from Jo2-induced apoptosis with significant expression of apoptosis- and antioxidant-related proteins in hepatocytes. (a) Apoptotic cell death of primary hepatocytes was quantitatively measured by ELISA. ${ }^{*} P<0.05$ vs. untreated, ${ }^{*} P<0.01$ vs. LacZ/Fas agonist. All data are expressed as mean $\pm \operatorname{SEM}(n=5)$. (b) Protein expression by Stat3-C in primary hepatocytes.

Stat3-C induces redox-related protein Ref-1. Because pretreatment with an antioxidant, NAC, partially improved Fas-mediated hepatic apoptosis (Figure 3c), we next investigated the expression of redox-related proteins in liver overexpressing Stat3-C. Protein expression of Ref-1 was remarkably upregulated by Stat3-C prior to Jo2 injection, though it was slightly downregulated by LacZ (Figure 4d). The increased amount of Ref- 1 protein was reduced following Jo 2 injection. In support of this, Stat3-C induced Ref-1 transcript 48-72 hours after AxCAS3-C injection (see Figure 4f), though it was not detected at all in livers injected with PS and AdLacZ. However, Stat3-C had no effect on the a

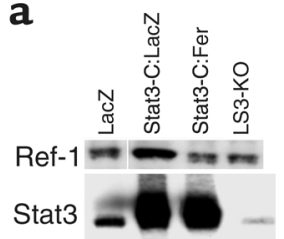

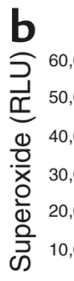
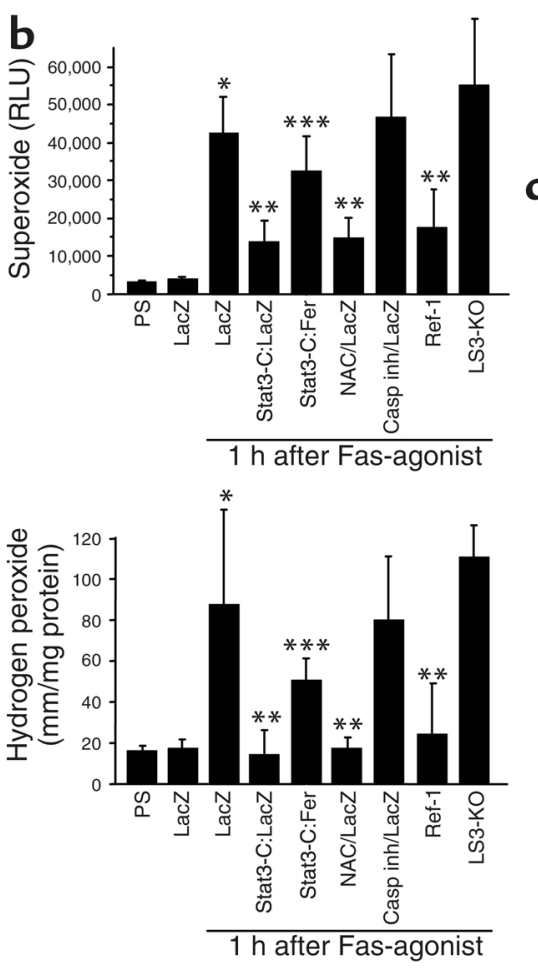

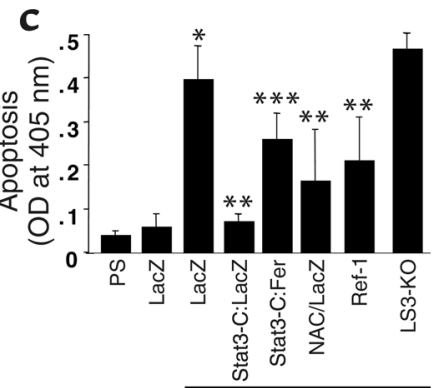

d $\mathrm{h}$ after Fas-agonist
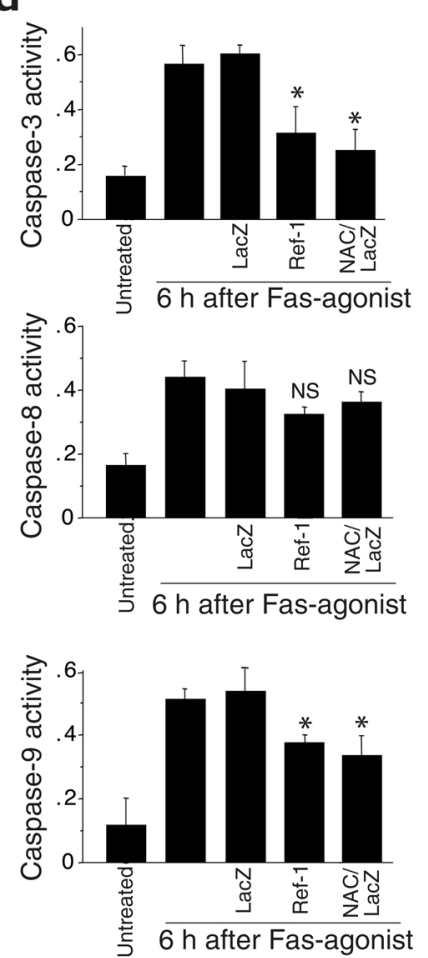
amounts of two other redox-related proteins, thioredoxin and MnSOD (Figure 4d). MnSOD was transiently increased after Jo2 injection in liver overexpressing Stat3-C, which may provide evidence for an oxidant-dependent mechanism in Fas-mediated liver injury. These findings indicate that activated Stat3 specifically upregulates Ref- 1 in mouse liver.

Transcriptional upregulation of proteins associated with Stat3. Ribonuclease protection assay revealed that, 48 hours after adenoviral gene transfer, the level of Fas mRNA was elevated by LacZ and Stat3-C, and $B c l-x L$, $B c l-2$, FLIP, and Ref- $1 \mathrm{mRNAs}$ were all elevated only by Stat3-C (Figure 4e). The level of FLICE mRNA, however, was not changed at all by LacZ and Stat3-C. We also confirmed Ref-1 mRNA expression by Northern blot analysis, because Ref-1 may play crucial roles in suppressing liver injury with its antioxidant and antiapoptotic properties (26). Stat3-C increased Ref-1 mRNA levels 48-72 hours after AxCAS3-C infection prior to Ref-1 protein expression (Figure $4 \mathrm{f}$ ), though it hardly detected in livers injected with PS and AdLacZ.

In order to see whether Ref- 1 is transcriptionally upregulated by Stat3-C, activation of Ref- 1 promoter by Stat 3 was also measured. Although AdLacZ moderately stimulated Ref-1 promoter activity, Stat3-C markedly stimulated the activity in a dose-dependent manner (Figure 4g).

Effects of Stat 3 upon primary hepatocytes. To examine whether Stat3-C directly targets hepatocytes or not, we performed experiments using primary cultured mouse hepatocytes. Exogenous Stat3-C was successfully expressed by infection of AxCAS3-C at an MOI of 2 in primary hepatocytes, and reduced Fas-induced apoptosis (Figure 5, a and b). Stat3-C treatment resulted in overexpression of Ref-1, FLIP, Bcl-2, and Bcl-xL proteins in primary hepatocytes as well as in liver. This indicates that Stat 3 directly targets at least hepatocytes and may have antiapoptotic and antioxidant effects upon hepatocytes.

Stat3-C reduces hepatic ROS production and apoptosis through Ref-1. Adenoviral vector encoding antisense fulllength Ref-1 gene (AdFer) was injected into the mice in order to investigate the role of Ref- 1 in the hepatic generation of ROS and Fas-mediated liver injury. The amount of Ref-1 protein was increased in Stat3-C mice coinfected with AdLacZ, but was reduced in Stat3-C mice coinfected with AdFer (Figure 6a). Hepatic ROS were produced 1 hour after Jo2 injection; these were reduced by overexpression of Stat $3-\mathrm{C}$ and Ref-1, and by pretreatment with NAC. ROS production, however, was not affected by caspase inhibitor alone. This antioxidant effect of Stat3-C was partially reversed by coinfection with AdFer. Stat3-C was considered to have an antioxidant effect through induction of Ref-1 (Figure 6b). Jo2-induced hepatic apoptosis was suppressed by pretreatment with NAC as well as Stat3-C. As expected, the suppression of apoptosis by Stat3-C was also reduced by AdFer coinfection (Figure 6c). Assays of caspase activities revealed that Ref- 1 and NAC equally reduced caspase- 3 activity and partially reduced caspase- 9 activity induced by Jo2 injection, but did not reduce caspase- 8 activity (Figure 6d). Ref- 1 may have reduced caspase activities (caspase- 3 and -9) by reducing oxidative stress. These facts suggest that Stat 3 possesses redox-dependent antiapoptotic properties through inactivation of caspase- 3 and -9 resulting from increased Ref-1 expression.

\section{Discussion}

This study reports on the protective effects of an activated form of Stat 3 against Fas-mediated liver injury and the underlying mechanisms of these effects (Figure 7). One of the major protective mechanisms against Fas-induced apoptosis in mouse liver seems to be a caspase-dependent mechanism. Stat3-C suppressed hepatic apoptosis through inhibition of both caspase- 8 and -3 activities via upregulation of FLIP protein and through upregulation of $\mathrm{Bcl}-2$ and $\mathrm{Bcl}-\mathrm{xL}$ proteins. The protective effect of Stat 3 against apoptosis has already been explained partly by upregulation of antiapoptotic proteins such as Bcl-xL and Survivin (13). IL-6, one of the major ligands for Stat3, is also reported to suppress liver injury of various causes (19, 33-35). Adenovirally overexpressed "hyper IL-6," a fusion protein of IL- 6 and its soluble receptor, reversed fulminant liver failure induced by D-galactosamine and also accelerated liver regeneration (35). IL-6 also inhibits Fas-mediated liver injury through upregulation of Bcl-2, Bcl-xL, and FLIP proteins (19). These reports may support our present data.

There are accumulating data indicating that ROS are involved in Fas-mediated apoptosis in various cells (36-39). In the present study, the antioxidant NAC also reduced hepatic generation of ROS and activity of caspase- 3 and -9 , and prevented the liver from injury

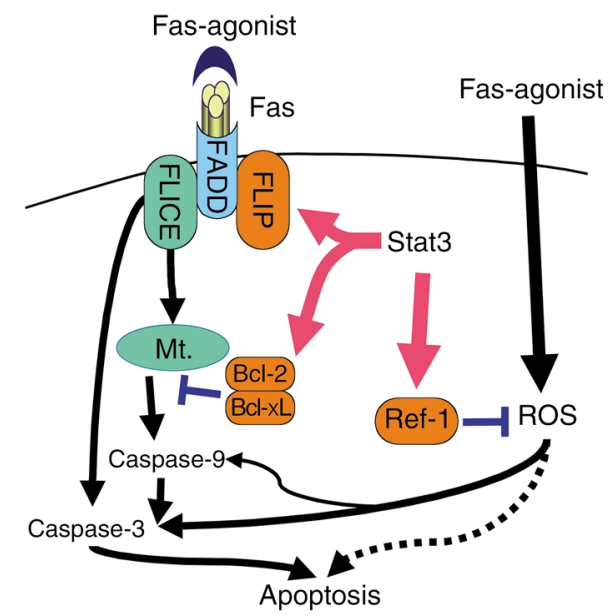

\section{Figure 7}

Schematic view of the protective mechanisms of Stat 3 against Fasmediated liver injury. Activation of Stat 3 upregulates caspase-related proteins (such as FLIP, Bcl-xL, and $\mathrm{Bcl}-2$ ) and redox-related protein Ref-1. These proteins collaboratively prevent Fas-mediated liver injury in redox-dependent and/or independent ways. Mt., mitochondria. 
induced by Fas agonist. Gulbins et al. have already reported the involvement of Ras-mediated ROS in Fasmediated cell death (40). Fas may activate caspase activities at least partially via ROS generation and inducing apoptosis, effects that are inhibited by NAC in this pathway.

Interestingly, Stat 3 reduced the hepatic generation of ROS following Jo2 administration through upregulation of Ref-1. Ref- 1 is primarily a nuclear protein with dual and mutually exclusive nuclear functions: it is an endonuclease in the base excision repair pathway (41) and it is a reducing agent that facilitates the DNAbinding activities of many redox-sensitive transcription factors including NF- $\mathrm{KB}(26,42-44)$. This is a bifunctional protein that is upregulated in response to a host of stimuli that result in intracellular ROS generation (45). Adenovirally overexpressed Ref- 1 in mouse liver successfully improves postischemic liver injury through suppression of ROS generation and apoptosis in hepatic tissue (26). This may also explain its role in the Stat3-dependent protection against Fas-induced oxidative liver injury through Ref- 1 . Stat3-C did not induce other redox-related proteins such as thioredoxin and MnSOD in the present study, though MnSOD induced by Stat 3 protects cardiomyocytes against hypoxia/reoxygenation-induced injury (46). In the present study, activities of caspase- 3 and caspase- 8 were both reduced by Stat3-C. The underlying mechanisms of this effect may involve the direct upregulation by Stat3-C of caspase-associated antiapoptotic proteins such as FLIP, Bcl-2, Bcl-xL, and also caspase-3 inactivation via upregulation of Ref- 1 .

In this way, Stat 3 may play crucial roles in protection against Fas-mediated liver injury. However, very interestingly, hepatic ROS generation and apoptosis were almost the same in control and LS3-KO mice. There may also exist in the cell compensatory mechanisms other than those dependent on Stat 3 that are not yet known.

PKB (Akt), a serine-threonine kinase, possesses mitogenic and antiapoptotic actions in endothelial cells when stimulated by HGF (47). Also, we have recently reported that Akt phosphorylates and inactivates the small GTPase Rac1, which results in the reduction of oxidative stress and apoptosis induced by hypoxia/ reoxygenation in hepatocytes (48). Ping et al. have reported that cardiac-specific transgenic activation of PKC- $\varepsilon$ confers cardioprotection to the ischemic heart (49). These data suggest that some mitogenic signaling molecules may possess antioxidant and/or antiapoptotic properties, and may also support our present data.

Stat 3 is one of the most important transcription factors and plays important roles in the initiation of liver regeneration. Though Stat3's effects upon cell cycle-related genes have been extensively studied, antiapoptotic effects and mechanisms of Stat 3 have not been well studied. This study is the first to report that Stat 3 protects against Fas-mediated hepatic apoptosis by its direct effects on caspase activity with antioxidant effects through Ref-1.

\section{Acknowledgments}

We are grateful to James E. Darnell for Stat3-C cDNA and for his critical review, to Seiichi Suzuki for encouragement, and to Mika Kamekawa for her excellent technique in immunohistochemical staining. We also thank Jurg Tschopp for mouse FLIP cDNA.

1. Darnell, J.E., Jr. 1997. STATs and gene regulation. Science. 277:1630-1635. 2. Schuringa, J.J., Jonk, L.J., Dokter, W.H., Vellenga, E., and Kruijer, W. 2000. Interleukin-6-induced STAT3 transactivation and Ser727 phosphorylation involves Vav, Rac-1 and the kinase SEK-1/MKK-4 as signal transduction components. Biochem. J. 347:89-96.

3. Leu, J.I., Crissey, M.A., Leu, J.P., Ciliberto, G., and Taub, R. 2001. Interleukin-6-induced STAT3 and AP-1 amplify hepatocyte nuclear factor 1mediated transactivation of hepatic genes, an adaptive response to liver injury. Mol. Cell. Biol. 21:414-424.

4. Schuringa, J.J., van der Schaaf, S., Vellenga, E., Eggen, B.J., and Kruijer, W. 2002. LIF-induced STAT3 signaling in murine versus human embryonal carcinoma (EC) cells. Exp. Cell Res. 274:119-129.

5. Levy, D.E., and Lee, C.K. 2002. What does Stat3 do? J. Clin. Invest. 109:1143-1148. doi:10.1172/JCI200215650.

6. Zhong, Z., Wen, Z., and Darnell, J.E. 1994. Stat3: a STAT family member activated by tyrosine phosphorylation in response to epidermal growth factor and interleukin-6. Science. 264:95-98.

7. Lutticken, C., et al. 1994. Association of transcription factor APRF and protein kinase Jak1 with the interleukin-6 signal transducer gp130. Science. 263:89-92.

8. Hirano, T., Ishihara, K., and Hibi, M. 2000. Roles of STAT3 in mediating the cell growth, differentiation and survival signals relayed through the IL-6 family of cytokine receptors. Oncogene. 19:2548-2556.

9. Bowman, T., Garcia, R., Turkson, J., and Jove, R. 2000. STATs in oncogenesis. Oncogene. 19:2474-2488.

10. Takeda, K., et al. 1997. Targeted disruption of the mouse Stat3 gene leads to early embryonic lethality. Proc. Natl. Acad. Sci. U. S. A. 94:3801-3804.

11. Li, W., Liang, X., Kellendonk, C., Poli, V., and Taub, R. 2002. STAT3 contributes to the mitogenic response of hepatocytes during liver regeneration. J. Biol. Chem. 277:28411-28417.

12. Debonera, F., et al. 2001. Activation of interleukin-6/STAT3 and liver regeneration following transplantation. J. Surg. Res. 96:289-295.

13. Shen, Y., Devgan, G., Darnell, J.E., and Bromberg, J.F. 2001. Constitutively activated Stat3 protects fibroblasts from serum withdrawal and UV-induced apoptosis and antagonizes the proapoptotic effects of activated Stat1. Proc. Natl. Acad. Sci. U. S. A. 98:1543-1548.

14. Grandis, J.R., et al. 2000. Constitutive activation of Stat 3 signaling abrogates apoptosis in squamous cell carcinogenesis in vivo. Proc. Natl. Acad. Sci. U. S. A. 97:4227-4232.

15. Takeda, K., et al. 1998. Stat3 activation is responsible for IL-6-dependent $\mathrm{T}$ cell proliferation through preventing apoptosis: generation and characterization of $\mathrm{T}$ cell-specific Stat3-deficient mice. J. Immunol. 161:4652-4660.

16. Nagata, S. 1999. Fas ligand-induced apoptosis. Annu. Rev. Genet. 33:29-55.

17. Matsumura, H., et al. 2000. Necrotic death pathway in Fas receptor signaling. J. Cell Biol. 151:1247-1256.

18. Siegmund, D., et al. 2001. Fas-associated death domain protein (FADD) and caspase- 8 mediate up-regulation of $\mathrm{c}$-Fos by Fas ligand and tumor necrosis factor-related apoptosis-inducing ligand (TRAIL) via a FLICE inhibitory protein (FLIP)-regulated pathway. J. Biol. Chem. 276:32585-32590.

19. Kovalovich, K., et al. 2001. Interleukin-6 protects against Fas-mediated death by establishing a critical level of anti-apoptotic hepatic proteins FLIP, Bcl-2, and Bcl-xL. J. Biol. Chem. 276:26605-26613.

20. Budd, R.C. 2002. Death receptors couple to both cell proliferation and apoptosis. J. Clin. Invest. 109:437-442. doi:10.1172/JCI200215077.

21. Pinkoski, M.J., Brunner, T., Green, D.R., and Lin, T. 2000. Fas and Fas ligand in gut and liver. Am. J. Physiol. Gastrointest. Liver Physiol. 278:G354-G366.

22. Galle, P.R., et al. 1995. Involvement of the CD95 (APO-1/Fas) receptor and ligand in liver damage. J. Exp. Med. 182:1223-1230.

23. Kanzler, S., and Galle, P.R. 2000. Apoptosis and the liver. Semin. Cancer Biol. 10:173-184.

24. Faubion, W.A., et al. 1999. Toxic bile salts induce rodent hepatocyte apoptosis via direct activation of Fas. J. Clin. Invest. 103:137-145.

25. Bromberg, J., and Darnell, J.E. 2000. The role of STATs in transcriptional control and their impact on cellular function. Oncogene. 19:2468-2473.

26. Ozaki, M., Suzuki, S., and Irani, K. 2002. Redox factor-1/APE suppresses oxidative stress by inhibiting the rac1 GTPase. FASEB J. 16:889-890.

27. Yamakawa, Y., et al. 2000. Interaction of platelet activating factor, reactive oxygen species generated by xanthine oxidase, and leukocytes in the 
generation of hepatic injury after shock/resuscitation. Ann. Surg. 231:387-398.

28. Daemen, M.A.R.C., et al. 1999. Inhibition of apoptosis induced by ischemia-reperfusion prevents inflammation. J. Clin. Invest. 104:541-549.

29. Yakar, S., et al. 1999. Normal growth and development in the absence of hepatic insulin-like growth factor I. Proc. Natl. Acad. Sci. U. S. A. 96:7324-7329.

30. Ozaki, M., et al. 2000. Inhibition of the Rac1 GTPase protects against nonlethal ischemia/reperfusion-induced necrosis and apoptosis in vivo. FASEB J. 14:418-429.

31. Grosch, S., Fritz, G., and Kaina, B. 1998. Apurinic endonuclease (Ref-1) is induced in mammalian cells by oxidative stress and involved in clastogenic adaptation. Cancer Res. 58:4410-4416.

32. Grosch, S., and Kaina, B. 1999. Transcriptional activation of apurinic/ apyrimidinic endonuclease (Ape, Ref-1) by oxidative stress requires CREB. Biochem. Biophys. Res. Commun. 261:859-863.

33. Galun, E., Zeira, E., Pappo, O., Peters, M., and Rose-John, S. 2000. Liver regeneration induced by a designer human IL-6/sIL-6R fusion protein reverses severe hepatocellular injury. FASEBJ. 14:1979-1987.

34. Peters, M., et al. 2000. Combined interleukin 6 and soluble interleukin 6 receptor accelerates murine liver regeneration. Gastroenterology. 119:1663-1671.

35 . Hecht, N., et al. 2001. Hyper-IL-6 gene therapy reverses fulminant hepatic failure. Mol. Ther. 3:683-687.

36. Malassagne, B., et al. 2001. The superoxide dismutase mimetic MnTBAP prevents Fas-induced acute liver failure in the mouse. Gastroenterology. 121:1451-1459.

37. Suzuki, Y., Ono, Y., and Hirabayashi, Y. 1998. Rapid and specific reactive oxygen species generation via NADPH oxidase activation during Fasmediated apoptosis. FEBS Lett. 425:209-212.

38. Giardina, C., Boulares, H., and Inan, M.S. 1999. NSAIDs and butyrate sensitize a human colorectal cancer cell line to TNF-alpha and Fas ligation: the role of reactive oxygen species. Biochim. Biophys. Acta. 1448:425-438.
39. Jayanthi, S., Ordonez, S., McCoy, M.T., and Cadet, J.L. 1999. Dual mechanism of Fas-induced cell death in neuroglioma cells: a role for reactive oxygen species. Brain Res. Mol. Brain Res. 72:158-165.

40. Gulbins, E., et al. 1996. Fas-induced programmed cell death is mediated by a Ras-regulated O2-synthesis. Immunology. 89:205-212.

41. Demple, B., Herman, T., and Chen, D. 1991. Cloning and expression of APE, the cDNA encoding the major human apurinic endonuclease: definition of family of DNA repair enzymes. Proc. Natl. Acad. Sci. U. S. A. 88:11450-11454.

42. Mitomo, K., et al. 1994. Two different cellular redox systems regulate the DNA-binding activity of the p50 subunit of NF-kappa B in vitro. Gene. 145:197-203.

43. Jayaraman, L., et al. 1997. Identification of redox/repair protein Ref-1 as a potent activator of p53. Genes Dev. 11:558-570.

44. Huang, L.E., Arany, Z., Livingston, D.M., and Bunn, H.F. 1996. Activation of hypoxia-inducible transcription factor depends primarily upon redoxsensitive stabilization of its alpha subunit. J. Biol. Chem. 271:32253-32259.

45. Nakamura, H., Nakamura, K., and Yodoi, J. 1997. Redox regulation of cellular activation. Annu. Rev. Immunol. 15:351-369.

46. Negoro, S., et al. 2001. Activation of signal transducer and activator of transcription 3 protects cardiomyocytes from hypoxia/reoxygenationinduced oxidative stress through the upregulation of manganese superoxide dismutase. Circulation. 104:979-981.

47. Nakagami, H., et al. 2001. Mitogenic and antiapoptotic actions of hepatocyte growth factor through ERK, STAT3, and AKT in endothelial cells. Hypertension. 37:581-586.

48. Ozaki, M., Haga, S., Zhang, H., Irani, K., and Suzuki, S. 2003. Inhibition of hypoxia/reoxygenation-induced oxidative stress in HGF-stimulated anti-apoptotic signaling: role of PI3-K and Akt kinase upon rac1. Cell Death Differ. 10:508-515.

49. Ping, P., et al. 2002. Formation of protein kinase CE-Lck signaling modules confers cardioprotection. J. Clin. Invest. 109:499-507. doi:10.1172/ JCI200213200. 